\title{
The spread and prediction of Ebola virus
}

\author{
Yingyou Chen,Mingzhe Wen,Minghao Geng \\ School of Public Health, Nanping Medical University, Fujian, China
}

\begin{abstract}
Ebola virus is a potent infectious disease virus that can cause Ebola haemorrhagic fever caused by human and primate. It has high mortality and easy infectivity to form a great obstacle to the steady development of human society. The profound understanding of the virus is particularly important harm. In this paper, a number of mathematical models are established to solve this problem. The software is used to analyze and predict the propagation of Ebola virus. The residual analysis is used to test the model. Finally, the effects of various control measures on controlling the epidemic are analyzed. In order to solve the problem, we will establish the infectious disease model to dynamically describe the spread of the virus in the 'virtual orangutan population'. Considering that the latent population is analyzed in this question, we will improve the model. Join the latent group (), and the migrants are divided into self-healing () and the dead (), to establish a suitable solution to this problem model. According to the relevant data given in the title, differential equations were established. For the second question, this question involves the one-way transmission of the virus across the species, so we can improve the model, on the basis of human contact with orangutans infected groups, the establishment of a one-way model to solve this problem. On the basis of the problem one, the differential equation is established, the model is predicted and tested. In the case of question 3, the number of human susceptible groups is much higher than that of the orangutan infection group by comparing the relevant data with the increase of the cure rate to $80 \%$ after the intervention of the outside experts. Therefore, the original data of human populations from experts can be ignored. Since then the virus spreads within a single species, the differential equation can be established according to the model in question 1 and the data values in the virtual human population are predicted. For question 4, the effect of the measures such as the strict enforcement of the various epidemic control measures and the improvement of the drug effect on the control of the epidemic are analyzed by comparing the above-mentioned models with the control measures.
\end{abstract}

Key words:model, model, differential equation, residual analysis,

\section{Problem restatement}

\subsection{Background}

Ebola virus (also translated as Ebola virus) is able to cause humans and primates to produce Ebola hemorrhagic fever of infectious diseases. A variety of non-human primates are generally susceptible, the virus latency is usually only 5 days to 10 days, 2 to 5 days after infection, fever, 6 to 9 days until death. 1 to 4 days after the onset until death, the blood contains the virus. Ebola virus infection in the mortality rate is between $50 \%$ to $90 \%$.

In the early stages of the disease, the Ebola virus may not be highly contagious, and during this period the patient may not even be infected, and as the disease progresses, the patient's body fluids due to diarrhea, vomiting and bleeding will have a high degree of biological risk; there seems to be born to Ebola immunized people, after the recovery of those who will invade them that kind of Ebola virus immunity.

Ebola virus is difficult to eradicate, so far there have been many outbreaks of the outbreak. According to Baidu Encyclopedia, the most recent one in 2014. As of September 25, 2014, the outbreak of Ebola in West Africa has led to more than 3,000 deaths, and another 6500 was diagnosed infection. Even more frightening thing is that the Ebola virus may be mutated through breathing!

\subsection{Formulation of the problem}

Suppose a region has 200,000 inhabitants and 3000 orangutans. People can be exposed to a certain probability of all the orangutans, when exposed to the orangutans that having the ability to spread, it must have a certain probability of infection with the virus, and human disease after the contact with the orangutans can be ignored. The incubation period between the man and the orangutan was two weeks. The researchers counted information such as the number of deaths and the number of deaths of humans and orangutans in the first 40 weeks (see Appendix 1). Please answer the following questions according to the relevant information:
1.2.1.According to the number of orangutans and the number of deaths, the establishment of a virus transmission model, and predict the dynamic description of the virus in the 'virtual orangutan population' in the spread and predict the next changes in the orangutan epidemic, and the following format gives 'virtual Orangutan population 'at week 80 , week 120 , week 200 ;

1.2.2.To establish a model of disease transmission of 'virtual population' mutual infection, to comprehensively describe the development of human and orangutan epidemics and to predict the development of the next epidemic in these two groups and to present the related data of 'virtual human population' in the following format Week 80, Week 120, Week 200

1.2.3.Assuming that at the 41 st week, outside experts begin to intervene and immediately control human contact with orangutans and raise the cure rate of the isolated treatment population to $80 \%$ through a specific drug. Please predict the future development of the 'virtual human population', compare the results of the second question to explain its role and impact, given the 'virtual human population' in the 45 weeks, 50 weeks, 55 weeks of the relevant data, The data format is same with the problem 2;

1.2.4.Please follow the above mathematical model to analyze the strict implementation of various epidemic control measures and the effects of drugs (including epidemic prevention drugs, quarantine drugs and therapeutic drugs, etc.) on the control of the epidemic.

\section{Problem Analysis}

2.1 To solve the thinking of the problem

Question one:

In order to solve the problem, this paper intends to establish an infectious disease model to dynamically describe the spread of the virus in the 'virtual orangutan population' and to predict the subsequent changes in the orangutan population. Taking into account the classic infectious disease model only susceptible to who are infecting, who are infected, out of those who carry out research, and this question requires the analysis of latent population, so we improve the model, on the basis of adding latent groups (), and Remove the person into the 
self-healing () and the dead (), the establishment of a model to solve the problem. According to the relevant data given in the title to establish differential equations, we use software to solve the forecast. Finally, the residual analysis is introduced to test the accuracy of the model.

Question 2:

On the basis of question two, on the basis of question 1, this question takes into account that the other species (orangutans) have a one-way virus transmission to the research species (human), so we can improve the model and add the infected groups that have already contacted with orangutans, the establishment of a one-way model to solve this problem. It is similar to the problem one, the establishment of differential equations, model prediction and testing.

Question 3:

For the third time, 41 weeks ago, orangutans and human had normal contact, the changes of the number in the virtual human applied to the one-way model; 41 weeks, due to the intervention of outside experts, human and orangutan contact has been strictly controlled, At this point we can predict according to the model of a model of virtual human population in the data values to predict.

Since the original data on the Ebola virus transmission in a single human population is not given, we have analyzed that the theoretical value of the latent (infected) is equal to the total number of known, minus the number of the orangutan spread of the virus and become latent The difference between the person (the infected person). However, according to the data in Appendix II and III, it is known that the number of human susceptible groups is much higher than that of orangutans, that is, the number of orangutans transmitted to humans is 40 years.

Question 4:

In response to question 4 , it is required to analyze the effects of various measures such as strict enforcement of various epidemic control measures and improvement of drug effects on the control of the epidemic. In this regard, we should compare it with the stage without any measures, and then the role of the object to the five groups in the model, and then to elaborate the role of measures.

\section{Model assumptions}

3.1. Assuming that the birth rate of the researcher during the forecast period has little effect on the population size;

3.2. Assuming that the orangutans and humans have a latency of one week, and one week after the latency will occur;

3.3. Assume that vulnerable people will be infected as latent once they are exposed to the infected person;

3.4. Assuming that the exit is no longer infected;

3.5. Assuming that the good heal in advance will not be disbanded.

\section{Symbol description}

Symbol Description

The population of orang utans and humans that are easily infected

The latent population of orang utan and humans

Infected amount among orang utans and humans

The number of orang utans or humans that self-healing

The amount of death that out of humans and orang utans

The number of people that isolated in humans

The daily growth rate of species A and species exposure groups

The ratio of latency group ( Species B exposed to Species A )

The infected ratio when species B exposed to species A

The daily exposure rate of the infected personDaily contact rate of infected beings

\author{
Rate of cure per-day \\ Rate of death per day \\ Incidence of latency \\ The rate of isolation per day
}

\section{Question one}

5.1 The establishment of the model

For this problem, create a model as shown below:

Figure 1 Model the population of virtual orangutan

The total number of orangutans is the same, the virtual orangutan population is divided into vulnerable, latent, infected, selfhealing, dead .

The daily contact rate of the infected person, the latency of the latent day, the daily cure rate, the daily mortality rate, the latent orangutan morbidity rate

From the above assumptions

$$
\text { (1) }
$$

The model is:

\subsection{Model Solving}

\subsubsection{Data processing}

According to Appendix I, it is easy to get the number of infected, dead and self-healing persons per week in the virtual orangutan population. As vulnerable people will be infected with infected people will be infected as a latent, and one week of orangutans incubation period, one week after the disease.

Thus, the number of latents this week is equal to the sum of the number of infected persons and the number of migrants next week minus the sum of the number of infected but not withdrawn by the end of the week,

Infectious persons are non-infected healthy orangutans in the virtual orangutan population, and data from 1-40 weeks of the orangutan population are compiled according to the relevant data in Appendix 1 (see Appendix II).

\subsubsection{Parameter calculation}

According to the established model, the parameters of the differential equation established are solved by using the relevant data:

Date of exposure to infection:

(3)

The rate of latent exposure per day :

The rate of cure per day:

Day mortality rate:

Incubate Orangutan Incidence :

Substituting the data in Appendix II into formulas (3) - (7), estimate:

$0, \quad 1.44 \mathrm{E}-3,0.0143,0.0286,0.5121$

After the parameters of the differential equation are obtained, the data of the eleventh week is selected as the initial value, that is, 
Using the differential equation to solve, predict the short-term ,,, the number of changes were as follows:

Figure 2 Orangutan susceptible predictor

Figure 3 Orangutan latent predictor

Figure 4 The predicts on infected orangutan

Figure 5 Orangutan Rescue Predictors

Figure 6 Predicts on death of orang utans

Figure 6 Predicts on death of orang utans

Through the above forecast results, the predicted values are as follows: Table 1:

Table 1 'Virtual Orangutan Population' Group Quantity Forecast Results (Unit: only)

Latent population In the onset healing Death due to illness

$\begin{array}{lllll}\text { Week 80 } & 5 & 10 & 297 & 411 \\ \text { Week 120 } & 1 & 2 & 322 & 445 \\ \text { Week 200 } & 0 & 0 & 327 & 452\end{array}$

\subsection{Analysis of results}

Through the analysis of Figure 2 - Figure 6 shows:

1.Predictable period of vulnerable, latent, infected people are basically the same trend, are in a short period of time the rapid decline eventually stabilized. However, in a biological group in the initial stage of the outbreak of the epidemic is often accompanied by a sharp rise in the number of infected and latent, it can be seen in the subject given the virtual orangutan population epidemic has been out for some time.

2. Because of the self-regulation ability of the later ecosystem, the epidemic can be controlled, and the risk of the infected person, the latent person and the susceptible person is declining and tends to be gentle. At the same time, the number of the self-healing person and the deathier is increasing and gradually becoming flat.

The results show that the epidemic is still in the serious stage, and in the period of about 130 weeks, the curve of each image tends to be flat. Infected, latent, infected, self-healing, the death of those who basically reached the stability, indicating that the epidemic is basically under control, orangutans no longer have Ebola virus infection is no longer infected with Ebola virus.

\subsection{Model test}

In the process of parameter solving, we validate the data given in the question, and the result is in good agreement with the actual data. In order to verify the correctness of the results and improve the accuracy of the model predictions, In the data and predictions given residual analysis, the results are as follows:

Figure 7 Permeable residuals

Figure 8 Latent residuals

Figure 9 residuals of infected persons

Figure 10 residuals of self-healers

Figure 11 Residual residuals

It can be seen from the five residual graphs that the residuals of the remaining data are close to zero except for the partial anomalies, and the confidence interval of the residuals contains zero. This explains the correctness of the model prediction.

5.5 Evaluation and promotion of models

5.5.1 Advantages of the model

1. The model is an improvement to the general model. The classification of the virtual epidemic population is more comprehensive. The establishment of the differential equation and the solution of the parameters are consistent with the virtual population, which laid a good foundation for the prediction of the late epidemic basis;

The residual analysis introduced in the model test intuitively evaluates the correctness and feasibility of the model prediction. Through its trend forecasting, it can provide reasonable suggestions for the epidemic researcher and provide the scientific basis for the control of the epidemic.

\subsubsection{The shortcomings of the model}

1. In order to simplify the problem, the parameters of this model are set to constant values, but in the virtual population contact rate , day cure rate, mortality, morbidity by a variety of factors common to difficult to constant.

2. The model does not consider the variation of Ebola virus itself in the process of transmission and the diversity of individuals within the population.

\subsubsection{Model promotion}

1. The model is suitable for a variety of epidemiological transmission predictions, for example, viruses 、;

2. The vigorous development of the financial industry has led to the rapid flow of funds, but also bring a strong financial crisis, this model can be extended to the banking and stock market crisis control, the appropriate use of the corresponding solution to solve the problem; 
3. The rapid development of Internet makes the dissemination of information and resources is very convenient, widely used in the social network today, its high-speed multi-channel social network information dissemination can apply the model to predict, control and solve related problems.

\section{Questions two}

6.1 Establishment of Unidirectional Model [4]

On the basis of question 1, the question of adding Ebola virus will also spread from the orangutan to the crowd this restriction, which can be spread the flow chart as follows:

Figure 12 Model of virtual orangutans and human populations

The unidirectional model of unidirectional transmission of Ebola virus between two species can be obtained by some improvement of the model, and the following differential equation can be established:

Which represents the daily growth rate of species and species exposure groups, indicating the species exposure to the species latent group ratio, indicating that the species exposed to the incidence of species infected group.

6.2 Model Solving

6.2.1 Data processing

Similar to the propagation law given in question 1 , we can calculate the number of potential human population latent this week, such as the next week, the next week, the death of the next week and the next week and the isolation and the next.

Whereby data from 1-40 weeks of human population are obtained from the relevant data in Appendix I (see appendix III).।

\subsubsection{Parameter calculation}

According to the established one-way model, the parameters of the differential equation are solved by using the relevant data:

The population of orang utans and humans that are easily infected

The latent population of orang utan and

humans

humans

self-healing

orang utans

species exposure

groups
The ratio of latency group ( Species B

Infected amount among orang utans and

The number of orang utans or humans that

The amount of death that out of humans and

The number of people that isolated in humans

The daily growth rate of species A and exposed to Species A )

The infected ratio when species B exposed to

species A
Rate of cure per-day :

(12)

Rate of death per day :

Incidence of latency :

The rate of isolation per day :

The daily growth rate of $\mathrm{B}$ species and A species exposure group:

The ratio of the $\mathrm{B}$ species to the latent population is: (17)

Indicating that the incidence of $\mathrm{B}$ species exposed to the infected group is:

(18)

(10) - (18) solution, the estimation:

$7.03503 * 10-6,=5.0057 * 10-4,=0.42250=1.11105$

$====-1.23907 * 10-7,=0.737784$

According to the parameters obtained, select the eleventh week as the initial data, namely:

$$
\text { , ,, }
$$

Into the differential equation (8) in the use of solution (procedures see Appendix IV):

Figure 13 Predator of Human Latent

Figure 14 Predicted value of human infection

Figure 15 Predictors of human self-healing

Figure 16 Predicted values of human deaths

Figure 17 Human isolator predictions

Through the above prediction, the forecast results are shown in the following table:

Table 2 'Virtual Human Population' Group Quantity Forecast Results (Unit:)

Latent Population In the onset Isolation treatment

Accumulated of cures Accumulated amount of death due to illness

$\begin{array}{llllll}\text { WEEK 80 } & 100 & 17 & 13 & 1266 & 3183 \\ \text { WEEK 120 } & 100 & 5 & 6 & 1435 & 3529 \\ \text { WEEK 200 } & 100 & 0 & 1 & 1435 & 3529\end{array}$

The daily exposure rate of the infected person

Daily contact rate of infected beings

Rate of cure per-day

Rate of death per day

Incidence of latency

The rate of isolation per day

The daily exposure rate of the infected person :

(10)

Daily contact rate of infected beings : 


\subsection{Results analysis}

As can be seen from Figure 13 - Figure 17:

1. The trend of the predictive value of the virus in the one-way propagation between the two populations is similar to the propagation of the virus in a single population. That is, the improved model of the model is applicable to the one-way propagation between the two populations. Thus, the model can be extended to multiple populations after the relevant conditions are flexibly transformed.

2. The number of isolators, the healers and the number of survivors increased, but eventually stabilized when the initial values were increased to about 100 weeks. The infected and latent curves decreased and decreased In the gentle, we can see that the epidemic is still in a serious stage;

3. In about 130 weeks, the image values tend to be stable, indicating that the epidemic is basically under control.

\subsection{Model test}

Similar to the model test method in question 1 , we introduce the residual analysis, using the program (see Appendix V) to verify the accuracy of the model established, the results are as follows:

Figure 18 People are vulnerable to residuals

Figure 19 Latent residuals

Figure 20 Residual human infection

Figure 21 Residual of human healers

Figure 21 Residency of human deaths

Figure 22 Residual of human isolators

It can be seen from the result graph that the residuals of the remaining data are close to zero except for the partial anomaly points, and the confidence interval of the residuals contains zero. This explains the correctness of the one-way model prediction.

6.5 Evaluation and promotion of models

6.5.1 Advantages of the model

1. Unidirectional model on the basis of the model to consider the contact with other species of infection, and the actual situation is more consistent. And one-way model is more practical than the model, more easily in agriculture, medicine, forestry to promote;

2. The model follows the test method of the model and its model, which ensures the correctness and consistency of the prediction result.

\subsubsection{The shortcomings of the model}

1. One-way model has added a new influencing factor to the improvement of the model, but it does not consider many random and uncertain factors that affect its own forecast.

2. This question only considers the unidirectional transmission of orangutans to human beings. However, in practice, the two species should be mutually influential.

\subsubsection{Model promotion}

The influence of the propagation of the unidirectional model species makes it more practical. Therefore, the prevention and control of the spread of pests and diseases and the prevention and control of other viruses in agriculture and forestry can be predicted by one-way model.

\section{Questions three}

7.1 Model establishment

On the basis of question two, the question strictly controlled the contact of humans with orangutans at 41 weeks. According to Appendix II, the relevant data of Appendix III shows that the number of human susceptible populations is much higher than that of orangutans, that is, the number of infections of orangutans to humans during 40 weeks is negligible. On the basis of model two to reduce the impact of orangutans on the crowd, which can be established differential equation is as follows:

The relevant parameters in the problem have been identified in the two, the direct use of the predicted 40 weeks after the epidemic in the 'virtual human population' in the development of the situation.

\subsection{Model Solving}

According to the relevant data in Appendix III and the procedure for the calculation of the parameters obtained in Question 2 (Appendix IV), the results are as follows:

Through the above prediction, the forecast results are shown in the following table:

Table 3 'Virtual Human Population' Group Forecast Results (Unit:)

Figure 23-27 The predicted values

Latent population In the onset Isolation treatment

Accumated of cure Death due to illness

$\begin{array}{clllll}\text { Week 45 } & 1104 & 90700 & 43710 & 60060 & 26170 \\ \text { Week 50 } & 29 & 30400 & 71280 & 97860 & 42710 \\ \text { Week 55 } & 1 & 3008 & 82950 & 11390 & 49710\end{array}$

\subsection{Results analysis}

By analyzing the graphs predicted by the above model and comparing it with the predictions of no control measures in question two, we can visually see that:

1. In the strict control of orangutans and human contact, the Ebola virus in the virtual human population in the spread of the problem and the spread of changes in the situation is very similar;

2. Compared with the problem 2, after the addition of isolation and treatment measures, due to the exit of the orangutan infected group makes the contact between the human vulnerable group and the infected group is relatively reduced, so the human population latent, the curve of the decline in the rate of infection. However, as mentioned earlier, the orangutan infected group is much less than the human susceptible group, so the isolation measures for the latent, the decline in the rate of infection is not the dominant factor;

3. The increase in the cure rate of the effects of drugs is directly affected by the infection, so that the increase in the rate of death of human populations is significantly slower than that in question 2 , and the growth rate of the cured patients is also accelerated. 
the actual value, similar to the problem of one or two of the model test, the introduction of residual analysis here, no longer tired here.

7.5 Evaluation of the model

Since this question only considers the spread of Ebola virus in a single species, we follow the model in question 1, the advantages of the model as mentioned above, here is no longer cumbersome.

7.5.1 The shortcomings of the model

In analyzing the problem, we neglected the effect of the first 40 weeks of orangutan populations on the initial population of human populations and the infected population. Although the number of orangutans in the order of magnitude is negligible, the orangutan infected group directly or indirectly affects the five quantitative changes in the model, whereby all the quantities in the population of the whole population will deviate from the final The data will have some error.

8 Questions four

Combined with the above analysis of the data we can see: the above three conditions only three problems in the epidemic to take preventive measures, so we can predict the data and the trend of the curve of the two or three questions to compare the situation, the following conclusions :

1. With the isolation measures, the exit of the orangutan infected group reduced the exposure of the human susceptible group to the infected group, resulting in a decrease in the rate of the latent and infection curve in the human population relative to the situation in question 2 This, from the implementation of measures on the spread of Ebola virus spread have a certain inhibitory effect;

2. The improvement in the cure rate of the effects of the three drugs in the problem III resulted in a downward trend in the number of death groups in the human population and a significant slowdown compared with the problem 2. Thus, the improvement of the therapeutic effect directly affected the population Mortality, the control of the epidemic plays a decisive role;

3. Through access to relevant information, anti-epidemic drugs, quarantine drugs were applied to vulnerable groups and latent groups, the improvement of these two kinds of drugs to make people more easily, earlier found, prevention and treatment of latent groups, thus, , The effect of quarantine drugs on the spread of Ebola virus spread also have a certain inhibitory effect.

\section{Reference}

[1] HOU Kuo, LI Shi-lin. Study on SIR model of influenza A H1N1 influenza transmission [J]. Journal of Hunan University of Technology. 24 (4): 41-42, 2010.

[2] Sun Hai, Xiong Sican, Wu Zhijiang. Study on Prevention and Control of Influenza A H1N1 Influenza Based on Improved SIR Model [J]. Journal of East China Institute of Technology. 37 (1): 97100, 2014.

[3] Jiang Qiyuan, Xie Jinxing, Ye Jun. Mathematical model [M].

Education Press.

[4]

Http://wenku.baidu.com/view/10aa898bbalaa8114531d902.html.

2015-08-7.

\section{REFERENCES}

[1] HOU Kuo, LI Shi-lin. Study on SIR model of influenza A H1N1 influenza transmission [J]. Journal of Hunan University of Technology. 24 (4): 41-42, 2010.

[2] Sun Hai, Xiong Sican, Wu Zhijiang. Study on Prevention and Control of Influenza A H1N1 Influenza Based on Improved SIR Model [J]. Journal of East China Institute of Technology. 37 (1): 97-100, 2014.

[3] Jiang Qiyuan, Xie Jinxing, Ye Jun. Mathematical model [M]. Beijing: Higher Education Press.

[4] Baidu library.

Http://wenku.baidu.com/view/10aa898bba1aa8114531d902.html. 2015-08-7. 